\title{
On Holomorphic Foliations Without Algebraic Solutions
}

\author{
S. C. Coutinho and Bruno F. M. Ribeiro
}

\section{CONTENTS}

1. Introduction

2. Holomorphic Foliations

3. Rational Exponents

4. The Examples

Acknowledgements

References

AMS Subject Classification: Primary, 13P10, 34A05, 34C05; Secondary, 58F18

Keywords: Gröbner bases, holomorphic foliation, algebraic solution
We give a symbolic algorithm to check whether certain holomorphic foliations of the complex projective plane have an algebraic solution. This algorithm is then used to produce new examples of foliations without algebraic solutions.

\section{INTRODUCTION}

The study of algebraic solutions of foliations on the complex projective plane goes back to G. Darboux [1878]. One of its main contributors in the nineteenth century was H. Poincaré; at the beginning of [Poincaré 1891], he says:

To find out if an equation of the first order and of the first degree is algebraically integrable, it is enough to find an upper bound for the degree of the integral; after this one need only perform some purely algebraic calculations.

A more recent milestone in the subject was J. P. Jouanolou's lecture notes [1979]. An often quoted result from these notes states that the set of holomorphic foliations of the complex projective plane $\mathbb{P}^{2}$ that do not have an algebraic solution is dense in the space that parametrizes the foliations; see [Jouanolou 1979, Chapter 4, p. 157 ff.]. In order to prove this result, Jouanolou had to construct a particular example of a foliation of $\mathbb{P}^{2}$ without algebraic solutions. However, both Jouanolou's and later proofs of this fact depend on the many special properties possessed by this example; see [Jouanolou 1979, p. 160 ff.; Lins Neto 1988, p. 219 ff.; Cerveau and Lins Neto 1991, p. 90]. Moreover, all the other explicit examples of holomorphic foliations of $\mathbb{P}^{2}$ without algebraic solutions that have been constructed are essentially variations on Jouanolou's. See Section 4 for more details. 
The purpose of this paper is to use the methods of computer algebra to search for new examples of foliations without algebraic solutions. The strategy consists in using the Gröbner bases method to automate Poincaré's 'purely algebraic calculations'. In this way we can write down an example of a foliation that may be expected to have no solutions and check whether that is so using the algorithm.

Of course, Poincaré's strategy depends on one being able to find an upper bound for the degree of the algebraic solution of a given foliation. Although such a bound does not always exist, a sharp bound is known for foliations with no positive rational exponents - see Section 2 for the definition. So we must first check whether a foliation is of this type, before we try to find an algebraic solution. Luckily the foliations that satisfy this condition form a dense set in the space that parametrizes holomorphic foliations of $\mathbb{P}^{2}$.

In Section 2 we describe the strategy behind the algorithm, and use results from the theory of holomorphic foliations to explain why it works. The part of the algorithm that checks that the foliation does not have positive rational exponents is discussed in Section 3. Finally, in Section 4 we give new examples of foliations of $\mathbb{P}^{2}$ without algebraic solutions. Unlike most other examples these are quite different from Jouanolou's foliation. To check that the examples satisfy the required properties we use an implementation of our algorithms in the computer algebra system Singular [Greuel et al. 1998].

\section{HOLOMORPHIC FOLIATIONS}

Let $\left[z_{0}: z_{1}: z_{2}\right]$ be homogeneous coordinates in the complex projective plane $\mathbb{P}^{2}$. A holomorphic foliation $\mathcal{F}$ in $\mathbb{P}^{2}$ is given by a 1 -form $\omega=\sum_{i=0}^{2} A_{i} d z_{i}$ where $A_{0}, A_{1}, A_{2}$ are homogeneous polynomials in $z_{0}, z_{1}, z_{2}$ of degree $k+1$ such that $\sum_{i=0}^{2} z_{i} A_{i}$ is identically zero. The singular set of $\mathcal{F}$ is the algebraic variety with equations $A_{i}=0$ for $0 \leq i \leq 2$. It will be denoted by $\operatorname{Sing} \mathcal{F}$.

Let $U_{i}$ be the open set of $\mathbb{P}^{2}$ defined by $z_{i} \neq 0$. Since $U_{0} \cong \mathbb{C}^{2}$, a vector field on $\mathbb{C}^{2}$ gives rise to a 1-form on $U_{0}$, and thus to another way of defining a foliation of $\mathbb{P}^{2}$. For let $x_{1}=z_{1} / z_{0}$ and $x_{2}=z_{2} / z_{0}$ be coordinates in $U_{0}$ and let $P_{1}$ and $P_{2}$ be polynomials in $\mathbb{C}\left[x_{1}, x_{2}\right]$ with no common factors. To the vector field $P_{1} \partial / \partial x_{1}+P_{2} \partial / \partial x_{2}$ there corresponds the 1 form $\alpha=P_{2} d x_{1}-P_{1} d x_{2}$ in the open set $U_{0}$. Denote by $\pi: \mathbb{C}^{3} \backslash\left\{z_{0}=0\right\} \rightarrow U_{0}$ the map

$$
\pi\left(z_{0}, z_{1}, z_{2}\right)=\left(z_{1} / z_{0}, z_{2} / z_{0}\right)=\left(x_{1}, x_{2}\right) .
$$

The pullback $\pi^{\star} \alpha$ is a form in $\mathbb{C}^{3}$ with poles in $z_{0}=$ 0 . Now choose $d$ so that $\omega=z_{0}^{d} \pi^{\star} \alpha$ is a 1 -form that is not divisible by $z_{0}$. It is easily checked that $\omega$ satisfies all the requirements of the first paragraph, so it determines a foliation of $\mathbb{P}^{2}$. Moreover, it is not difficult to show that all foliations of $\mathbb{P}^{2}$ can be defined in this way.

Of course there is nothing special about $U_{0}$, and the same construction holds equally well for both $U_{1}$ and $U_{2}$. However, for the sake of consistency we will assume that the foliations in this paper are always defined by vector fields on $U_{0}$.

Convention. Let $\mathcal{F}$ be a foliation of $\mathbb{P}^{2}$. Throughout the paper the notation $\mathcal{F}=\mathcal{F}\left(P_{1}, P_{2}\right)$ means that:

(1) $P_{1}, P_{2}$ are polynomials of $\mathbb{C}\left[x_{1}, x_{2}\right]$ with no common factors, and

(2) $\mathcal{F}$ is defined in $U_{0}$ by the vector field $P_{1} \partial / \partial x_{1}+$ $P_{2} \partial / \partial x_{2}$.

We must define what it means for an algebraic curve $S$ of $\mathbb{P}^{2}$ to be invariant under $\mathcal{F}$. Without loss of generality we may assume that $S \cap U_{0} \neq \varnothing$, and that it is the set of zeroes in $U_{0}$ of a polynomial $f \in$ $\mathbb{C}\left[x_{1}, x_{2}\right]$. Let $\mathcal{F}=\mathcal{F}\left(P_{1}, P_{2}\right)$. Then $S$ is invariant under $\mathcal{F}$ when there exists a polynomial $g \in \mathbb{C}\left[x_{1}, x_{2}\right]$ such that

$$
P_{1} \partial f / \partial x_{1}+P_{2} \partial f / \partial x_{2}=g f .
$$

In this case we also say that $S$ is an algebraic solution of $\mathcal{F}$.

Now let $z \in \mathbb{P}^{2}$ be a singular point of $\mathcal{F}$. As in the previous paragraph, we can assume that $z \in U_{0}$ and that $\mathcal{F}=\mathcal{F}\left(P_{1}, P_{2}\right)$. Then $P_{1}(z)=P_{2}(z)=0$. Denote by $J_{\mathcal{F}}(z)$ the jacobian of $\left(P_{1}, P_{2}\right)$ in $z$. If the eigenvalues of $J_{\mathcal{F}}(z)$ are nonzero then $\mathcal{F}$ is said to be nondegenerate at $z$, and we can compute their ratios. Following Poincaré's terminology [1891] we call these ratios the exponents of the singularity. A nondegenerate foliation is one which is nondegenerate at all of its singular points. The set of all exponents of a nondegenerate foliation $\mathcal{F}$ will be denoted by $\operatorname{Exp}(\mathcal{F})$. 
We retain, for the moment, the notation of the previous paragraph. Denote by $k(\mathcal{F})$ the greater of $\operatorname{deg} P_{1}$ and $\operatorname{deg} P_{2}$, and by $P_{i}^{(j)}$ the homogeneous components of degree $j$ of the polynomial $P_{i}$. If $k=k(\mathcal{F})$, let

$$
\Delta_{i}(\mathcal{F})=x_{2} P_{1}^{(k-i)}-x_{1} P_{2}^{(k-i)} .
$$

These polynomials play a key rôle in the next theorem, which is at the core of the algorithms we propose. We will denote the line $z_{0}=0$ of $\mathbb{P}^{2}$ by $L_{\infty}$.

Theorem. Let $\mathcal{F}$ be a nondegenerate foliation of $\mathbb{P}^{2}$.

(1) The line $L_{\infty}$ is invariant under $\mathcal{F}$ if and only if $\Delta_{0}(\mathcal{F})$ is a nonzero polynomial.

(2) If $\Delta_{0}(\mathcal{F})$ is identically zero and $\operatorname{Exp}(\mathcal{F}) \cap \mathbb{Q}^{+}=\varnothing$ then any irreducible algebraic curve of $\mathbb{P}^{2}$ invariant under $\mathcal{F}$ must have degree less than or equal to $k(\mathcal{F})$.

The proof of this theorem follows by collating a number of known results in the theory of holomorphic foliations. We give a proof here both for the sake of completeness, and because some of the formulae will be required for our algorithm. For a proof with a more algebraic-geometric bent, see [Bernstein and Lunts 1988, Appendix, theorem 4, p. 241].

In the proof of the theorem we use the degree of a foliation; its definition and properties can be found in [Lins Neto 1988, Section 1.4].

Proof. Let $\mathcal{F}=\mathcal{F}\left(P_{1}, P_{2}\right)$. In order to simplify the notation we will write $k$ for $k(\mathcal{F})$ and $\Delta_{i}$ for $\Delta_{i}(\mathcal{F})$ throughout the proof.

Let $y_{1}=z_{0} / z_{1}$ and $y_{2}=z_{2} / z_{1}$ in $U_{1}$. In these coordinates the line $L_{\infty}$ has equation $y_{1}=0$. Moreover, in $U_{0} \cap U_{1}$ we have $x_{1}=1 / y_{1}$ and $x_{2}=y_{2} / y_{1}$. Performing this change of variables in the 1-form $P_{1} d x_{2}-P_{2} d x_{1}$ and multiplying the resulting form by $y_{1}^{k+2}$ to cancel the pole, we obtain

$$
y_{1} \hat{P}_{1} d y_{2}-\left(y_{2} \hat{P}_{1}-\hat{P}_{2}\right) d y_{1}
$$

where $\hat{P}_{i}\left(y_{1}, y_{2}\right)=y_{1}^{k} P_{i}\left(1 / y_{1}, y_{2} / y_{1}\right)$. The coefficient of degree $j$ of $\hat{P}_{i}$ considered as a polynomial in $y_{1}$ with coefficients in $\mathbb{C}\left[y_{2}\right]$ is $P_{i}^{(k-j)}\left(1, y_{2}\right)$. Thus

$$
y_{2} \hat{P}_{1}-\hat{P}_{2}=\Delta_{0}\left(1, y_{2}\right)+y_{1} \Delta_{1}\left(1, y_{2}\right)+y_{1}^{2} S
$$

for some polynomial $S \in \mathbb{C}\left[x_{1}, x_{2}\right]$.
Suppose first that $\Delta_{0}$ is a nonzero polynomial. Then $\Delta_{0}\left(1, y_{2}\right)$ is also nonzero, because $\Delta_{0}$ is homogeneous in $x_{1}$ and $x_{2}$. In particular, $y_{1}$ does not divide $y_{2} \hat{P}_{1}-\hat{P}_{2}$. Thus $\mathcal{F}$ is defined in $U_{1}$ by the vector field $y_{1} \hat{P}_{1} \partial / \partial y_{1}+\left(y_{2} \hat{P}_{1}-\hat{P}_{2}\right) \partial / \partial y_{2}$, so that $y_{1}=0$ is invariant under $\mathcal{F}$.

On the other hand, if $\Delta_{0}=0$ then we can cancel $y_{1}$ from $(2-2)$ so that $\mathcal{F}$ is represented in $U_{1}$ by the vector field

$$
\hat{P}_{1} \partial / \partial y_{1}+\left(\Delta_{1}\left(1, y_{2}\right)+y_{1} S\right) \partial / \partial y_{2}
$$

Now the coefficient of $\hat{P}_{1}$ of degree zero in $y_{1}$ is $P_{1}^{(k)}\left(1, y_{2}\right)$. But $y_{2} P_{1}^{(k)}=P_{2}^{(k)} \neq 0$, so that $\hat{P}_{1}$ is not divisible by $y_{1}$. Hence $y_{1}=0$ is not a solution of $\mathcal{F}$ which completes the proof of (1).

We now turn to $(2)$. If $\Delta_{0}(\mathcal{F})=0$ then $\mathcal{F}$ is a foliation of degree $k-1$ by [Lins Neto 1988, Lemma 2, p. 203]. Let $S$ be an irreducible algebraic curve of $\mathbb{P}^{2}$ invariant under $\mathcal{F}$. Since $\operatorname{Exp}(\mathcal{F}) \cap \mathbb{Q}^{+}=\varnothing$, it follows from [Soares 1993, Lemma 5.1, p. 156] that if $S$ is not smooth then all its singularities are normal crossings. Finally, by [Cerveau and Lins Neto 1991, theorem 1, p. 891] we have that the degree of such a curve $S$ must be smaller than or equal to the degree of the foliation plus 1 . Hence $\operatorname{deg} S \leq k$ and the proof is complete.

Corollary. We retain the notation and hypotheses of the main theorem. Suppose that $\Delta_{0}(\mathcal{F})=0$. Then:

(1) both $P_{1}$ and $P_{2}$ have degree exactly $k(\mathcal{F})$;

(2) the singularities of $\mathcal{F}$ in $L_{\infty} \cap U_{1}$ are given by

$$
P_{1}^{(k)}\left(1, y_{2}\right)=\Delta_{1}\left(1, y_{2}\right)=0
$$

(3) The trace and the determinant of the jacobian of $\mathcal{F}$ at a point $\left(0, y_{2}\right) \in U_{1} \cap \operatorname{Sing} \mathcal{F}$ are given by $t_{1}\left(1, y_{2}\right)$ and $d_{1}\left(1, y_{2}\right)$, respectively, where

$$
\begin{aligned}
& t_{1}\left(x_{1}, x_{2}\right)=\Delta_{2}+\partial P_{1}^{(k-1)} / \partial x_{2}, \\
& d_{1}\left(x_{1}, x_{2}\right)=\Delta_{2} \partial P_{1}^{(k)} / \partial x_{2}-P_{1}^{(k-1)} \partial \Delta_{1} / \partial x_{2} .
\end{aligned}
$$

(4) $[0: 0: 1]$ is a singular point of $\mathcal{F}$ if and only if $P_{2}^{(k)}(0,1)=\Delta_{1}(0,1)=0$. Moreover, if $[0: 0: 1] \epsilon$ Sing $\mathcal{F}$ then the trace and the determinant of the jacobian at this point are given by $t_{2}(0,1)$ and $d_{2}(0,1)$, respectively, where

$$
\begin{aligned}
t_{2}\left(x_{1}, x_{2}\right) & =\partial \Delta_{1} / \partial x_{1}-P_{2}^{(k-1)} \\
d_{2}\left(x_{1}, x_{2}\right) & =\Delta_{2} \partial P_{2}^{(k)} / \partial x_{1}-P_{2}^{(k-1)} \partial \Delta_{1} / \partial x_{1} .
\end{aligned}
$$


Proof. Let $k(\mathcal{F})=\max \left\{\operatorname{deg} P_{1}, \operatorname{deg} P_{2}\right\}$. Clearly if $\Delta_{0}=x_{2} P_{1}^{(k)}-x_{1} P_{2}^{(k)}$ is identically zero then both $P_{1}$ and $P_{2}$ must have degree $k$, thus proving (1). Since $\Delta_{0}=0$, it follows from equation $(2-3)$ that a singular point of $\mathcal{F}$ in $L_{\infty} \cap U_{1}$ must satisfy the equations

$$
P_{1}^{k}\left(1, y_{2}\right)=\Delta_{1}\left(1, y_{2}\right)=0
$$

which proves (2). The formulae in (3) follow from the computation of the partial derivatives of $\hat{P}_{1}$ and $\Delta_{1}\left(1, y_{2}\right)+y_{1} S$ at $\left(0, y_{2}\right)$. Note that except possibly for $[0: 0: 1]$, all the singular points of $\mathcal{F}$ at $L_{\infty}$ belong to $U_{1}$. Finally, (4) follows from an argument analogous to the above, applied to $[0: 0: 1] \in U_{2}$.

The main theorem suggests the following strategy for checking whether a given foliation has invariant algebraic curves:

First stage: Check whether $\mathcal{F}$ is a nondegenerate foliation with no positive rational exponents. If it is not, then we cannot be certain that the third stage will work properly.

Second stage: Check whether $\Delta_{0}(\mathcal{F})$ is zero. If it is nonzero, $L_{\infty}$ is invariant under $\mathcal{F}$; if zero, proceed to the third stage.

Third stage: Let $\mathcal{F}=\mathcal{F}\left(P_{1}, P_{2}\right)$. Check whether there is a nonconstant polynomial $f$ of degree $d$, where $0<$ $d \leq k(\mathcal{F})$, and a polynomial $g$ of degree $k(\mathcal{F})-1$ such that equation $(2-1)$ holds. If no such polynomials exist then $\mathcal{F}$ has no invariant curves.

The second stage is completely straightforward, and the third stage is also easy to implement. We use the naïve approach which is already found in [Darboux 1878, Section 2, p. $71 \mathrm{ff}$.]. It consists in choosing generic polynomials for $f$ and $g$, and equating coefficients on both sides of (2-1). Gröbner bases can then be used to check whether the system of equations admits a solution.

For the next algorithm it will be necessary to choose a term order in the set of monomials in $x_{1}$ and $x_{2}$ for which the constants have degree zero. We will make use of the usual multi-index notation, so that if $\alpha=\left(\alpha_{1}, \alpha_{2}\right) \in \mathbb{N}^{2}$ then $x^{\alpha}=x_{1}^{\alpha_{1}} x_{2}^{\alpha_{2}}$.

\section{Solution Algorithm}

Input: polynomials $P_{1}$ and $P_{2}$ of $\mathbb{C}\left[x_{1}, x_{2}\right]$.
Output: the degree of an algebraic solution of the foliation $\mathcal{F}\left(P_{1}, P_{2}\right)$, or 0 if $\mathcal{F}$ does not have a solution of degree less than or equal to $k(\mathcal{F})$.

1. Let $k=\max \left\{\operatorname{deg} P_{1}, \operatorname{deg} P_{2}\right\}$ and compute $\Delta_{0}=$ $x_{2} P_{1}^{(k)}-x_{1} P_{2}^{(k)}$. If $\Delta_{0} \neq 0$ return 1 .

2. Let $f=\sum_{|\alpha| \leq k} u_{\alpha} x^{\alpha}$ and $g=\sum_{|\beta| \leq k-1} v_{\beta} x^{\beta}$ and construct the ideal $I$ generated by the polynomials in the $u$ s and $v$ s that are the coefficients of the monomials $x^{\alpha}$ in $P_{1} \partial f / \partial x_{1}+P_{2} \partial f / \partial x_{2}-g f$.

3. Let $\mathcal{U}=\left\{x^{\alpha}:|\alpha| \leq k\right\}$.

4. Choose the element $x^{\alpha}$ of highest order in $\mathcal{U}$, and let $J_{\mathcal{U}}$ be the ideal generated by $I$ and $u_{\alpha}-1$.

5. If $1 \notin J_{\mathcal{U}}$ then return $|\alpha|$ and stop.

6. Add $u_{\alpha}$ to the generators of $I$, remove $x^{\alpha}$ from $\mathcal{U}$ and go back to step 4 .

Two important points about the algorithm should be observed. First, we can make $u_{\alpha}=1$ in step 4 because equation $(2-1)$ is homogeneous in the $u$ s. Second, since $f$ represents a constant polynomial when $\mathcal{U}=\{1\}$, it follows that $J_{\mathcal{U}} \neq(1)$ in this case. In particular the algorithm always stops.

Of course the algorithm merely searchs for an algebraic solution with degree less than or equal to the degree of the foliation. However, if the foliation has positive rational exponents then these may not exhaust all the possible solutions.

Since we are using Gröbner bases only to check whether a given ideal is trivial or not, we can choose any term order we want for this algorithm. In the next section we show how Gröbner bases can be used to handle the first stage of the strategy sketched above.

Finally, it has been shown in [Carnicer 1994] that an upper bound on the degree of an algebraic solution in terms of the degree of the foliation also exists for the more general case of a nondicritical foliation. It would be very interesting to find a way of using Gröbner bases to check nondicriticalness. For the definition see [Carnicer 1994; Cano 1988]; be warned that this is not quite the same as the 'classical' nondicritical condition.

\section{RATIONAL EXPONENTS}

The purpose of this section is to show how Gröbner bases can be used to implement the first stage of 
the strategy sketched in Section 2. We will sum up the various steps by which this is achieved in the algorithm at the end of the section.

Let $x_{1}=z_{1} / z_{0}$ and $x_{2}=z_{2} / z_{0}$ be coordinates in the open set $U_{0}$ of $\mathbb{P}^{2}$, and let $\mathcal{F}=\mathcal{F}\left(P_{1}, P_{2}\right)$ as in the conventions of Section 2. Let $k=k(\mathcal{F})=$ $\max \left\{\operatorname{deg} P_{1}, \operatorname{deg} P_{2}\right\}$ and $\Delta_{0}=\Delta_{0}(\mathcal{F})=x_{2} P_{1}^{(k)}-$ $x_{1} P_{2}^{(k)}$. As in Section 2, we will denote by $J_{\mathcal{F}}$ the jacobian matrix of $\left(P_{1}, P_{2}\right)$. Thus $\operatorname{tr} J_{\mathcal{F}}$ and $\operatorname{det} J_{\mathcal{F}}$ are both polynomials in $\mathbb{C}\left[x_{1}, x_{2}\right]$. We will consider separately the singularities in $U_{0}$, and those in $L_{\infty}$.

Suppose first that $u \in U_{0}$ is a singularity of $\mathcal{F}$. The worst case occurs when $u$ is a degenerate singular point; that is, when one of the eigenvalues of $J_{\mathcal{F}}(u)$ is zero. To find out if there are any degenerate singular points it is enough to compute a Gröbner basis for the ideal generated by $P_{1}, P_{2}$ and $\operatorname{det} J_{\mathcal{F}}$. If the basis is not equal to $\{1\}$, then $\mathcal{F}$ has degenerate singularities, and some of its exponents will not even be well-defined.

So we may assume, from now on, that $\mathcal{F}$ has no degenerate singular points in $U_{0}$. Let $\lambda_{1}$ and $\lambda_{2}$ be the eigenvalues of $J_{\mathcal{F}}(u)$ at some singular point $u \in U_{0}$ of $\mathcal{F}$. Since we are assuming that both eigenvalues are nonzero, it follows that the exponent $\eta=\lambda_{1} / \lambda_{2}$ is a well-defined complex number. Now

$$
\frac{\operatorname{tr}\left(J_{\mathcal{F}}(u)\right)^{2}}{\operatorname{det} J_{\mathcal{F}}(u)}=\frac{\left(\lambda_{1}+\lambda_{2}\right)^{2}}{\lambda_{1} \lambda_{2}}=\eta+\frac{1}{\eta}+2 .
$$

Thus, if

$$
\begin{aligned}
& \theta_{0}\left(x_{1}, x_{2}, s\right) \\
& \quad=\left(\operatorname{det} J_{\mathcal{F}}\right) s^{2}+\left(2 \operatorname{det} J_{\mathcal{F}}-\left(\operatorname{tr} J_{\mathcal{F}}\right)^{2}\right) s+\operatorname{det} J_{\mathcal{F}},
\end{aligned}
$$

and $u=\left(u_{1}, u_{2}\right)$, then $\theta_{0}\left(u_{1}, u_{2}, \eta\right)=0$.

The calculations above suggest that we should compute a Gröbner basis $G$ of the ideal generated by the polynomials $P_{1}, P_{2}$ and $\theta_{0}$ with respect to the lexicographical order that has $s<x_{1}<x_{2}$. Since the polynomials $P_{1}$ and $P_{2}$ have no common factor, the system $P_{1}=P_{2}=0$ has only finitely many solutions. Hence the same must hold for $P_{1}=P_{2}=$ $\theta_{0}=0$. By [Adams and Loustaunau 1994, Corollary 2.2 .11$, p. 65$]$ there must be a polynomial $e_{0}(s)$ in $G$ that contains only the variable $s$. In particular, if a complex number is an exponent of a singular point of $\mathcal{F}$ in $U_{0}$ then it is a root of $e_{0}(s)$. Thus if $e_{0}(s)$ has no positive rational roots, then $\mathcal{F}$ has no posi- tive rational exponents at those of its singularities that belong to $U_{0}$.

This settles the problem so far as singularities in $U_{0}$ are concerned. But, of course, $\mathcal{F}$ could have singularities in $L_{\infty}$. One way to deal with these is to repeat all the above for the vector fields that represent $\mathcal{F}$ in $U_{1}$ and in $U_{2}$. However, since the computations of a Gröbner bases under the lexicographical order can be rather slow it is convenient to use the formulae in the corollary of Section 2 to speed up the calculations.

First note that, by the corollary, $\mathcal{F}$ has singularities in $L_{\infty} \cap U_{1}$ if and only if the polynomials $P_{1}^{(k)}\left(1, y_{2}\right)$ and $\Delta_{1}\left(1, y_{2}\right)$ have a common root. Since these are polynomials in one variable, we can check whether they are coprime using the Euclidean algorithm.

Assuming that the polynomials have a common root, we must find whether $\mathcal{F}$ has degenerate singularities on $L_{\infty} \cap U_{1}$ and, if not, whether there are any positive rational exponents. The general strategy is the same as the one used for singularities in $U_{0}$, and more details can be found in the description of the algorithm given below. The rôle of $\theta_{0}$ is played, in this case, by the polynomial

$$
\begin{aligned}
& \theta_{1}\left(y_{2}, s\right) \\
& =d_{1}\left(1, y_{2}\right) s^{2}+\left(2 d_{1}\left(1, y_{2}\right)-t_{1}\left(1, y_{2}\right)^{2}\right) s+d_{1}\left(1, y_{2}\right),
\end{aligned}
$$

where $t_{1}\left(x_{1}, x_{2}\right)$ and $d_{1}\left(x_{1}, x_{2}\right)$ are defined in (3) of the corollary of Section 2. Finally, we must determine whether $[0: 0: 1]$ is a singularity of $\mathcal{F}$, and compute its exponent if it is nondegenerate. To do this we use (4) of the corollary of Section 2 just as we used (2) and (3) for the singularities in $L_{\infty} \cap U_{1}$.

Next we translate these ideas into an algorithm which determines whether a given foliation has any positive rational exponents.

\section{Rational Exponents Algorithm}

Input: polynomials $P_{1}$ and $P_{2}$ of $\mathbb{C}\left[x_{1}, x_{2}\right]$.

Output: true if $\mathcal{F}\left(P_{1}, P_{2}\right)$ has no positive rational exponents and false otherwise.

1. Use Gröbner bases to check whether the ideal generated by $P_{1}, P_{2}$ and $\operatorname{det} J_{\mathcal{F}}$ contains 1 . If it does not return false and stop.

2. Define $\theta_{0}\left(x_{1}, x_{2}, s\right)$ as above; then use Gröbner bases with respect to the lexicographical order 
$s<x_{1}<x_{2}$ to find a polynomial $e_{0}(s)$ in the variable $s$ alone, that belongs to the ideal generated by $P_{1}, P_{2}$ and $\theta_{0}$.

3. Check whether $e_{0}(s)$ has a positive rational root. If it does, return false.

4. Compute $g\left(y_{2}\right)=\operatorname{gcd}\left(P_{1}^{(k)}\left(1, y_{2}\right), \Delta_{1}\left(1, y_{2}\right)\right)$.

5. If $g=1$ return true; otherwise compute

$$
\operatorname{gcd}\left(g\left(y_{2}\right), d_{1}\left(1, y_{2}\right)\right)
$$

(see the corollary of section 2 for the definition of $d_{1}$ ). If it is not 1 , return false.

6. Define $\theta_{1}\left(y_{2}, s\right)$ as above, and use Gröbner bases, with respect to the lexicographical order $s<y_{2}$, to compute a polynomial $e_{1}(s)$ on the variable $s$ alone, that belongs to the ideal generated by

$$
P_{1}^{(k)}\left(1, y_{2}\right), \quad \Delta_{1}\left(1, y_{2}\right) \text { and } \theta_{1}\left(y_{2}, s\right) .
$$

7. Check whether $e_{1}(s)$ has a positive rational root; if it does, return false.

8. Compute $P_{2}^{(k)}(0,1)$ and $\Delta_{1}(0,1)$. If one of them is nonzero, return true; but if both are zero and $d_{2}(0,1)=0$, return false.

9. Compute

$$
\begin{aligned}
& \theta_{2}(s) \\
& \quad=d_{2}(0,1) s^{2}+\left(2 d_{2}(0,1)-t_{2}(0,1)^{2}\right) s+d_{2}(0,1) .
\end{aligned}
$$

If $\theta_{2}(s)$ has a positive rational root, return false; otherwise, return true.

When implementing this algorithm it is a good idea to check first whether the system $P_{1}=P_{2}=0$ has dimension zero or not. Of course if it does not, then the polynomials do not determine a foliation in the sense of Section 2.

Our implementation of this algorithm using the computer algebra system Singular has two peculiarities that should be mentioned. First, to check whether the one variable polynomials $e_{0}(s)$ and $e_{1}(s)$ have a positive rational root we first factorize them using the Singular command factorize. Second, when computing Gröbner basis with respect to the lexicographical order we use the Singular command stdfglm to speed up the calculations; see [Greuel et al. 1998, p. 236] and [Adams and Loustaunau 1994, pp. 67 and 68, exercises 2.2.7 and 2.2.8] for the algorithm behind this command.

\section{THE EXAMPLES}

Although it is known that the set of foliations of $\mathbb{P}^{2}$ without algebraic solutions is dense, a search of the literature reveals a dearth of concrete examples. In fact, all examples are variations of the Jouanolou foliation $[1979$, p. $160 \mathrm{ff}$.$] . A very interesting fam-$ ily of foliations of this sort is the one discovered by Żołądek [1998]. Unlike the foliations considered in this paper, the foliations in Żołądek's family can have degenerate singularities for some choice of parameters.

However, both Jouanolou's foliation and the nondegenerate foliations in the examples of Żoładek are of Poincaré type; that is, none of their exponents are positive real numbers. In fact, in all these examples the exponents of the nondegenerate foliations are not even real numbers. For the relevance of the Poincaré hypothesis to this problem, see [Lins Neto 1988] or [Cerveau and Lins Neto 1991].

In this section we give an example of a simple foliation that we have shown to have no algebraic solutions using our implementation of the algorithms solution and rational exponents in the computer algebra system Singular. It turns out that, unlike the previously known examples, this foliation has exponents that are positive real numbers; so they are not of Poincaré type.

Let

$$
\begin{aligned}
& P_{1}=2 x_{1}^{3}+3 x_{1}^{2} x_{2}+a x_{2}^{2}+1, \\
& P_{2}=2 x_{1}^{2} x_{2}+3 x_{1} x_{2}^{2}+b x_{1}^{2}+1,
\end{aligned}
$$

where $a b \neq 0$ and $8 a+27 b \neq 0$. Using Gröbner bases and the method of undetermined coefficients one shows that $x_{1} P_{2}-x_{2} P_{1}$ is an irreducible polynomial of degree 3 if $a \neq b$. In particular $\operatorname{gcd}\left(P_{1}, P_{2}\right)=1$, and we get a family of foliations $\mathcal{F}\left(P_{1}, P_{2}\right)$. Since this family is parametrized by $a$ and $b$ we will denote it by $\mathcal{F}(a, b)$ for short. One readily checks that $\Delta_{0}(\mathcal{F}(a, b))=0$ and that $\Delta_{1}(\mathcal{F}(a, b))=a x_{2}^{3}-b x_{1}^{3}$. In particular, the line at infinity is not invariant under $\mathcal{F}$. Moreover, the system

$$
\begin{gathered}
P_{1}^{(k)}\left(1, y_{2}\right)=2+3 y_{2}=0 \\
\Delta_{1}\left(1, y_{2}\right)=a y_{2}^{3}-b=0
\end{gathered}
$$

does not have a solution because $8 a+27 b \neq 0$. Since we also have that $\Delta_{1}(0,1)=a \neq 0$, we conclude that Sing $\mathcal{F} \subset U_{0}$. 
Let $I=\left\{(a, b) \in \mathbb{Z}^{2}: a \neq b\right.$ and $\left.1 \leq a, b \leq 10\right\}$. Using a computer and the rational exponents algorithm it takes less than a minute to check that for $(a, b) \in I$ the foliation $\mathcal{F}(a, b)$ has a rational exponent only when $a=9$ and $b=4$. However, even in this case the exponent is -1 , a negative rational number. On the other hand, using a combination of Gröbner bases and numerical computations in Maple V.5, we have shown that if $(a, b) \in I$ and $\mathcal{F}(a, b)$ has a positive real exponent then either $b=1$, or $a=3$ and $b=2$. Moreover all these real exponents occur at a real singularity. Finally, using an implementation of the solutions algorithm in Singular it is easy to check that $\mathcal{F}(a, b)$ does not have an algebraic solution for any $(a, b) \in I$.

We illustrate the output of the algorithms when $a=2$ and $b=3$. In the notation used to describe the rational exponents algorithm, we have

$$
\begin{aligned}
e_{0}(s)= & 757707876887 s^{14}-1515415753774 s^{13} \\
& -1582513020691 s^{12}-6026827836972 s^{11} \\
& +11777436443567 s^{10}+29426173550670 s^{9} \\
& +5040018620749 s^{8}-81748114201576 s^{7} \\
& +5040018620749 s^{6}+29426173550670 s^{5} \\
& +11777436443567 s^{4}-6026827836972 s^{3} \\
& -1582513020691 s^{2}-1515415753774 s \\
& +757707876887 .
\end{aligned}
$$

This polynomial is irreducible over $\mathbb{Q}$, so it has no rational roots. Using the solution algorithm we find that there is no invariant curve of degree less than or equal to 3 . Hence by the theorem of Section 2 we conclude that $\mathcal{F}(2,3)$ has no algebraic solutions.

Now, it turns out that $e_{0}(s)$ has two positive real roots, namely $0.7386877578 \ldots$ and its reciprocal. Although all exponents of $\mathcal{F}(2,3)$ are roots of $e_{0}(s)$, the converse need not be true. However, a numerical computation shows that one of the singularities of $\mathcal{F}(2,3)$ has real coordinates, and these roots turn out to be the two exponents of this singularity. In view of this, it seems reasonable to pose two questions:

- Are there any nonzero complex numbers $a$ and $b$, for which $(a-b)(8 a+27 b) \neq 0$ and $\mathcal{F}(a, b)$ has an algebraic solution?

- Is the set of all $(a, b)$ for which $\mathcal{F}(a, b)$ has a real exponent dense in $\mathbb{C}^{2}$ ?

\section{ACKNOWLEDGEMENTS}

We are grateful to Bruno Scárdua, Alcides Lins Neto and Jorge Vitório Pereira for their suggestions. We thank CNPq (Brazil) and PRONEX (commutative algebra and algebraic geometry) for financial support.

\section{REFERENCES}

[Adams and Loustaunau 1994] W. W. Adams and P. Loustaunau, An introduction to Gröbner bases, Graduate Studies in Math. 3, Amer. Math. Soc., Providence, RI, 1994.

[Bernstein and Lunts 1988] J. Bernstein and V. Lunts, "On nonholonomic irreducible $D$-modules", Invent. Math. 94:2 (1988), 223-243.

[Cano 1988] F. Cano, "Dicriticalness of a singular foliation", pp. 73-94 in Holomorphic dynamics (Mexico, 1986), edited by X. Gomez-Mont et al., Lecture Notes in Math. 1345, Springer, Berlin, 1988.

[Carnicer 1994] M. M. Carnicer, "The Poincaré problem in the nondicritical case", Ann. of Math. (2) 140:2 (1994), 289-294.

[Cerveau and Lins Neto 1991] D. Cerveau and A. Lins Neto, "Holomorphic foliations in $\mathbb{C P}(2)$ having an invariant algebraic curve", Ann. Inst. Fourier (Grenoble) 41:4 (1991), 883-903.

[Darboux 1878] G. Darboux, "Mémoire sur les équations différentielles algébriques du $\mathrm{I}^{\circ}$ ordre et du premier degré", Bull. des Sci. Math. (Mélanges) (1878), 6096, 123-144, 151-200.

[Greuel et al. 1998] G.-M. Greuel, G. Pfister, and H. Schönemann, "Singular version 1.2 User Manual", Report on Computer Algebra 21, Centre for Algebra, University of Kaiserslautern, 1998. See http:// www.mathematik.uni-kl.de/ zca/Singular.

[Jouanolou 1979] J. P. Jouanolou, Équations de Pfaff algébriques, Lect. Notes in Math. 708, Springer, Berlin, 1979.

[Lins Neto 1988] A. Lins Neto, "Algebraic solutions of polynomial differential equations and foliations in dimension two", pp. 192-232 in Holomorphic dynamics (Mexico, 1986), edited by X. Gomez-Mont et al., Lecture Notes in Math. 1345, Springer, Berlin, 1988.

[Poincaré 1891] H. Poincaré, "Sur l'integration algébrique des équations differentielles du $1^{\mathrm{er}}$ ordre", Rendiconti Circ. Mat. Palermo 11 (1891), 193-239. Reprinted in his Oeuvres, t. III, 35-58. 
[Soares 1993] M. G. Soares, "On algebraic sets invariant by one-dimensional foliations on $\mathbb{C P}(3)$ ", Ann. Inst. Fourier (Grenoble) 43:1 (1993), 143-162.
[Żołạdek 1998] H. Żołądek, "New examples of holomorphic foliations without algebraic leaves", Studia Math. 131:2 (1998), 137-142.

S. C. Coutinho, Departamento de Ciência da Computação, Instituto de Matemática, Universidade Federal do Rio de Janeiro, P.O. Box 68530, 21945-970 Rio de Janeiro, RJ, Brazil (collier@impa.br)

Bruno F. M. Ribeiro, Departamento de Ciência da Computação, Instituto de Matemática, Universidade Federal do Rio de Janeiro, P.O. Box 68530, 21945-970 Rio de Janeiro, RJ, Brazil (bruno@land.ufrj.br)

Received Received April 3, 2000; accepted March 26, 2001 